\title{
LA CREATIVIDAD ¿ABDUCTIVA? DE LA MEMORIA
}

\author{
ABDUCTIVE? CREATIVITY OF THE MEMORY
}

\author{
Antonio Duarte* \\ Universidad Complutense de Madrid, \\ Madrid - España. \\ Recibido junio de 2019/Received June, 2019 \\ Aceptado julio de 2019/Accepted July, 2019
}

\begin{abstract}
RESUMEN
En este artículo abordaré algunos aspectos relacionados con la creatividad de la memoria en conexión con lo que el filósofo Charles Sanders Peirce denominó abducción. Se mostrará que el acceso a los recuerdos en casos de memoria episódica puede reconstruirse en un marco abductivo. Este análisis revelará cómo el carácter falible del proceso abductivo desaparece cuando es nuestra memoria la que nos proporciona "ideas" (recuerdos) creativos, es decir, no fieles a la realidad.
\end{abstract}

Palabras Clave: Abducción, creatividad, memoria episódica, Peirce.

\begin{abstract}
Here I focus on some aspects related to the creativity of the memory in connection with what the philosopher Charles Sanders Peirce called abduction. In cases of episodic memory, the access to memories may be reconstructed following an abductive framework. In this analysis, I would like to highlight how the fallibility character of the abductive processes disappears when our memory provides us with creative "ideas" (memories), i.e. not faithful to reality.
\end{abstract}

Key Words: Abduction, creativity, episodic memory, Peirce.

\section{INTRODUCCIÓN}

En este artículo abordaré algunos aspectos relacionados con la creatividad de la memoria en conexión con lo que el filósofo Charles Sanders Peirce denominó abducción. En torno a la cuestión de la memoria podríamos hablar de que, en las últimas décadas, se ha producido (o se está produciendo) un cambio de paradigma, en el sentido de que estamos empezando a aceptar los fallos de nuestra memoria, no sólo en el sentido de recuerdos olvidados, sino también (y aquí se encuentra el cambio de paradigma) en la creciente evidencia de que, en diversas ocasiones, nuestra memoria nos proporciona recuerdos inventados (ver, por ejemplo, Loftus \& Ketcham, 2010; Manzanero \& Álvarez, 2015). Por otro lado, también en las últimas décadas, se está produciendo un rescate de la abducción, convirtiéndose en nuestros días en un tema epistemológico de primer orden (Hintikka,

* Autor correspondiente / Corresponding author: antduart@ucm.es 
1998) dentro del proceso de construir y seleccionar hipótesis, ya sea en el terreno científico, artístico o cotidiano. Estableciendo la conexión entre estos dos terrenos aparentemente dispares se tratará de mostrar que el acceso a los recuerdos en casos de memoria episódica, puede reconstruirse en un marco abductivo, de acuerdo con la formulación de Peirce.

En este análisis, además, se pondrá de relieve cómo el carácter falible del proceso abductivo desaparece cuando es nuestra memoria la que nos proporciona "ideas" (recuerdos) creativas, es decir, no fieles a la realidad. Para llegar a este punto, abordaremos, en primer lugar el tema de la abducción enfatizando ciertas características de este tipo de razonamiento que serán importantes a la hora de realizar una analogía con la forma en que la memoria "crea" recuerdos (Sección 2). Tras esta descripción del razonamiento abductivo analizaremos algunos casos específicos dentro del vasto y complejísimo mundo de la memoria (Sección 3), con el fin de poder trazar las correspondencias existentes con la abducción. Las principales conclusiones de este estudio serán expuestas y discutidas en las Secciones 4 y 5.

\section{CONSIDERACIONES EN TORNO A LA ABDUCCIÓN}

\subsection{Teoría de la abducción de Peirce}

Charles Sanders Peirce desarrolló durante más de cincuenta años su teoría de la abducción (ver, por ejemplo, Fann, 1970; Santaella, 2005; Aliseda, 2006; Gabbay, Nossum, \& Woods, 2006). En esta sección se desarrollará una visión general de este tipo de razonamiento enfatizando ciertas características que serán importantes a la hora de realizar una analogía con la forma en que la memoria "crea" recuerdos.

La inferencia abductiva consiste básicamente en fabricar hipótesis. En este tipo de razonamiento (entendido en su forma silogística) la conclusión es una hipótesis: "la hipótesis se da cuando encontramos alguna circunstancia curiosa que podría ser explicada si fuera el caso particular de una cierta regla general, y es por eso que adoptamos tal suposición" (Peirce, 1878, CP 2.624 / Peirce, 2004, p. 2533, [traducción propia]). Peirce, más adelante, en On the Logic of Drawing History from Ancient Documents Especially from Testimonies, nos proporciona la primera afirmación completa de la teoría final de la abducción. Cuando nos encontramos ante acontecimientos sorprendentes buscamos una explicación:

La explicación debe ser una proposición tal que nos conduzca a la predicción de los hechos observados, como consecuencia necesaria o al menos como muy probable en esas circunstancias. Una hipótesis será adoptada entonces porque es probable en sí misma y porque interpreta los hechos con cierta probabilidad. Este paso de adoptar una hipótesis sugerida por los hechos es lo que yo llamo abducción. (Peirce, 1901,CP 7.202 / Peirce, 2004, p. 4532, [traducción propia]).

La forma específica de la inferencia abductiva sería la siguiente:

Se observa el hecho sorprendente C;

Aunque si A fuera cierto, $\mathrm{C}$ sería algo corriente,

Por lo tanto, hay razón para sospechar que A es cierto. (Peirce, 1903, CP 5.189/ Peirce, 2004, p. 3794, [traducción propia]).

En esta inferencia la aceptación de la hipótesis, y de todo el silogismo por tanto, es provisional. Esta provisionalidad nos conduce a la búsqueda de explicaciones alternativas cuando nos hallamos ante "alguna circunstancia curiosa". Debido a esta naturaleza de la inferencia abductiva, Peirce estableció un método de selección de hipótesis a través de los tres estadios de la investigación surgidos a través de los tres tipos de inferencias (Peirce, 1901, CP 7.162-7.233 / Peirce, 2004, p. 4508-4552).

La abducción sería el primer paso de la investigación. Tras la adopción de la hipótesis habría que trazar sus consecuencias experimentales, este sería el segundo paso de la investigación, una deducción. El último caso consistiría en la verificación de la hipótesis comprobando las predicciones deducidas a partir de la hipótesis con resultados experimentales. Si estas coinciden, la hipótesis será seleccionada en ese punto de la investigación. Esta última inferencia que, basada en una hipótesis, prueba las predicciones mediante experimentos $\mathrm{u}$ observaciones, es a la que llamamos inducción. Así, los procesos abductivos llevan asociados tanto la generación como la selección de la hipótesis.

En resumen, la abducción sería el primer paso que damos a la hora de postular hipótesis, 
cuando nos enfrentamos a un hecho sorprendente. El lugar, en definitiva, donde acontece la creatividad: "la abducción es el proceso de formar una hipótesis explicativa. Es la única operación lógica que introduce una nueva idea" (Peirce, 1903, CP 5.171 / Peirce, 2004, p. 3786, [traducción propia]). La sorpresa, de la que se hace cargo la hipótesis, produce cierta desazón, cierta incredulidad, y ese estado de insatisfacción es desde el que demandamos buscar una abducción que convierta el hecho o fenómeno sorprendente en uno razonable. En todo caso la explicación abductiva no anula por completo esta irritación, tan solo la suaviza, en tanto en cuanto esta sugerencia requiere ponerse a prueba antes de convertirse en creencia (para un desarrollo de la teoría de la abducción en relación con las nociones peirceanas de hábito, duda y creencia, ver Nubiola, 2005). Gabbay y Woods (2005), en su modelo abductivo, sostienen que la abducción es un problema de preservación de ignorancia (o mitigación de la ignorancia), pero nunca "resolución" de ignorancia; esto es, mediante la abducción la ignorancia básica, que no ha de considerarse una total ignorancia, ni se soluciona ni se deja intacta. En contraposición con el carácter de preservación de ignorancia de la abducción, la deducción tendría un carácter de preservación de la verdad, y en la inducción el problema sería el de mejora probabilística. Por lo tanto, el razonamiento abductivo se da en respuesta a un problema de ignorancia donde uno tiene un propósito cognitivo que no puede resolver atendiendo a lo que sabe en ese momento (Magnani, 2015, p. 286). De esta manera, a través de la abducción se ofrece una explicación a un hecho sorprendente en forma de hipótesis. Esta hipótesis explica de alguna manera los datos observados. Sin embargo, la abducción ocurre en circunstancias abiertas y siempre surge como una explicación tentativa que puede modificarse a la luz de nuevas observaciones.

A partir de lo dicho, hay tres rasgos de la abducción que me gustaría destacar, tres rasgos que tratarán de relacionarse posteriormente con la creatividad de la memoria:

(i) su reestructuración de los elementos para normalizar un hecho sorprendente: los datos observados que parecían no tener conexión y, por tanto, causaban sorpresa, son explicados mediante de la hipótesis, (ii) su momento de insight: el momento en el que se percibe la solución al puzle o enigma cuando se da con una hipótesis plausible ante ese problema, y

(iii) su carácter falible, tentativo y sujeto a nuevos descubrimientos: la abducción suele ocurrir en circunstancias abiertas, cuando nuestro conocimiento es incompleto.

\subsection{Los procesos abductivos}

Es indudable que nuestro cerebro construye la realidad, pudiéndose afirmar por tanto que todo nuestro conocimiento se construye a través de hipótesis confirmadas y refinadas por la inducción (Peirce, 1901, MS 692 / Peirce, 1966). No hay, sin embargo, tal cosa como la "abducción pura", eso que Nietzsche, con su habitual ironía, llamaba el "dogma de la inmaculada percepción". En este sentido, cualquier percepción visual, táctil, olfativa... viene mediada también por un juicio perceptivo. Lo que caracteriza este tipo de percepciones, cuando todo va según lo previsto, es que se trata de un juicio "absolutamente forzado a mi aceptación" que se da en un proceso que "soy totalmente incapaz de controlar y [que], consecuentemente, soy incapaz de criticar" (Peirce, 1903, CP 5.157 / Peirce, 2004, p. 3779, [traducción propia]). Nuestro sistema visual, por ejemplo, hace continuas suposiciones. La percepción implica, en este aspecto, resolver problemas de ambigüedad. Digamos que rellenamos los huecos de las escenas visuales que nuestro cerebro no puede procesar.

Creo que para ilustrar este punto puede ser instructivo el siguiente ejemplo de narrativa:

1. María sujetaba fuertemente la cuerda de su bonito globo.

2. De repente, un fuerte viento se lo llevó.

3. El viento lo condujo a un árbol.

4. El globo golpeó una rama y explotó.

5. María lloró sin consuelo. (Walton, 2004,

p. 66, [traducción propia]).

Este es un ejemplo de narrativa anclada en el sentido común. Existen muchas premisas no explícitas y, sin embargo, todos rellenamos los huecos, inconscientemente, de la misma manera. Está claro que, en parte, estas cinco afirmaciones tienen sentido como conjunto porque nosotros asumimos como implícitas los así llamados entimemas (ver, por ejemplo, Walton, 2008): el todo es mayor que las partes. Por ejemplo, puede sugerirse 
que el viento causó que el globo se escapara de las manos de María pero, ¿no sujetaba María el globo fuertemente? Como afirma Walton (2004, p. 67), un abogado defensor del viento en un juicio habría alegado que las afirmaciones uno y dos son contradictorias. De hecho, María pudo soltar la cuerda voluntariamente, tras lo cual se lo llevó el viento. En la frase 4 parece sugerirse que el globo explotó porque golpeó una rama, pero nada indica que haya tal relación causal. Podría haber explotado porque un niño travieso lo disparó con su tirachinas. Del mismo modo en la frase quinta se dice que María lloró, y asumimos que fue debido a la pérdida del globo. Desde luego, parece que rige aquí la ley de la buena continuidad descrita por los psicólogos de la Gestalt alemana, ese proceso por el cual el cerebro hace que las cosas parezcan completas a partir de información incompleta.

Es el caso también de lo que ocurre en relación al ojo humano: la capa de fotorreceptores de la retina tiene un agujero a través del cual los nervios ópticos salen de la retina para transmitir la información al cerebro. Como el agujero carece de fotorreceptores no es posible ver los objetos que serían procesados en esa región. Si miramos con un ojo cerrado no vemos ese espacio vacío correspondiente al punto ciego. Nuestro cerebro llena el espacio vacío con una conjetura, con una conjetura, y eso es lo importante, que se nos impone.

Por supuesto, hay un salto cualitativo entre la imaginación creadora y esta tarea de rellenar huecos. No obstante, una experiencia incompleta o anómala es, precisamente, la que dispara el razonamiento abductivo.

Tratemos ahora de comparar estas experiencias basadas en juicios perceptivos y aquellas que están más cerca de la creatividad más volitiva e imaginativa. Entre los múltiples ejemplos que se podrían poner aquí, me centraré en un caso de invención fecunda que parece cumplir con los rasgos antes mencionados. Me refiero al caso del sabio griego Eratóstenes de Cirene, que vivió en el siglo III a. C. (276-194 a.C.), en concreto, a su cálculo de la circunferencia de la Tierra.

Un día, siendo director de la gran Biblioteca de Alejandría, leyó Eratóstenes en un papiro que en el mediodía del solsticio de verano en Siena (hoy Asuán, en Egipto) un palo vertical no proyectaba sombra alguna, que las sombras de las columnas del templo también desaparecían y que podía verse el sol reflejado en el fondo de un pozo hondo. Como afirma Carl Sagan: "Era una observación que otros podrían haber ignorado con facilidad. Palos, sombras, reflejos en pozos, la posición del Sol: ¿qué importancia podían tener cosas tan sencillas y cotidianas?" (Sagan, 1983, p. 14). Sin embargo, Eratóstenes halló cierta sorpresa al tener conocimiento de aquellos datos. Es decir, el papiro actuó como un detonador abductivo (Aliseda, 2005, 2006). No se puede contar la historia como si se tratara de un descubrimiento casual y como que Eratóstenes dio con la solución a partir de ciertos datos. La invención fecunda no surge ex nihilo. Presupone un contexto previo de trabajo y una voluntad de resolver un misterio, un problema o una carencia. El insight, el chispazo, siempre se da en el seno de un proceso creador racional, a causa de un foco teórico previo, si se quiere.

Según las observaciones registradas en el papiro, se deducía que el sol se hallaba en el cénit, el punto más alto en el cielo, en Siena el día del solsticio de verano al mediodía local. Eratóstenes experimentó qué ocurría en Alejandría en ese mismo momento, y constató que en Alejandría un palo vertical sí proyectaba sombra al mediodía del día 21 de junio, concluyendo que el sol se hallaba desplazado 7,2 grados con respecto al cénit. Para explicar aquella discrepancia "Eratóstenes comprendió que la única respuesta posible es que la superficie de la Tierra está curvada" (Sagan, 1983, p. 14). Por tanto, utilizando esa información y completándola con la distancia entre Siena y Alejandría obtuvo la circunferencia de la Tierra. Supuso que Siena y Alejandría tenían la misma longitud (realmente distan 3 grados) y que el Sol se encontraba a tal distancia de la Tierra que sus rayos podrían considerarse paralelos y midió la distancia entre Alejandría y Siena $(787,5 \mathrm{~km}$, 5.000 estadios), calculando que si 7,2 grados de circunferencia se corresponden con la distancia entre Alejandría y Siena $(787,5 \mathrm{~km})$, entonces una circunferencia completa de $360^{\circ}$ sería 787,5 x $(360 / 7,2)=39.375 \mathrm{~km}$. El error en la determinación de la circunferencia fue solo del 1,5\%, mínimo para una medición hecha hace 2.400 años.

No hay que olvidar que también se atribuye a Eratóstenes la invención de la esfera armilar con la que calculó un valor casi exacto del ángulo del eje de la Tierra con respecto al plano que forma está en su movimiento alrededor del Sol. Esto representó la determinación de la oblicuidad de la eclíptica. 
Es importante destacar que sus conocimientos de geografía y astronomía fueron básicos para llegar a estos descubrimientos.

En este punto me gustaría enfatizar que no hay tal inspiración súbita, descontextualizada. La teoría de la gravitación de Newton no habría sido posible sin las contribuciones anteriores de Kepler, Galileo y Huygens, el cálculo de fluxiones (necesario para la comprobación de la teoría), ni sin las propias tentativas anteriores del propio Newton. No obstante, uno de los elementos que quería destacar dentro de la abducción es, precisamente, la conexión entre hechos o sucesos aparentemente distantes o inconexos que permite reorganizar la forma de abordar un hecho sorprendente. Esta capacidad para conectar dos hechos aparentemente inconexos, en este caso la incidencia de los rayos del sol y la circunferencia de la Tierra, son los casos de abducciones en el extremo más creativo del espectro. Entonces, lo que podríamos llamar el relámpago mágico o la iluminación repentina se produce dentro de un contexto y, además, la propia emoción, el Eureka, no garantiza la autenticidad de la hipótesis sin su comprobación posterior, siempre sujeta a nuevos descubrimientos. De esta forma, al contrario de lo que ocurre en los juicios perceptivos, somos capaces de someter a crítica nuestras conjeturas.

El propio Einstein lo expresaba de forma muy clara cuando cierto día de la década de 1930 invitó a Saint-John Perse a Princeton para averiguar cómo trabaja el poeta:

“¿Cómo surge la idea de un poema?" Le preguntó. El poeta le habló del papel que desempeña la intuición y la imaginación. "Lo mismo le ocurre al hombre de ciencia", respondió Einstein encantado. "Es una iluminación repentina, casi un éxtasis. Es cierto que luego la inteligencia analiza y los experimentos confirman o invalidan la intuición. Pero, inicialmente, se produce un gran salto de la imaginación". (Isaacson, 2009, p. 592).

En este relato vemos recogidas gran parte de las ideas que quiero poner aquí en relación: la intuición creadora, que se manifiesta a partir del insight; la imaginación (o "razón creativa", Barrena, 2008) como forma de conectar esos acontecimientos que previamente estaban, por decirlo de algún modo, aislados unos de otros; y la falibilidad, en último término, de esa intuición que, lejos de manifestarse con una intuición pura sería, en todo caso, una intuición contextualizada. El propio Peirce se oponía al intuicionismo y no se puede encontrar en ningún lugar de sus escritos que afirmara que la abducción fuera intuitiva. Alejémonos, pues, de la noción común de la intuición como conocimiento infalible e inmediato de la realidad: la intuición abductiva, si así quiere llamarse, daría cabida tanto a la teoría de Herbert Spencer y a la teoría del flogisto, por ejemplo, como a las suposiciones abductivas de Kepler o Newton. Un intuicionista stricto sensu no admitiría las primeras; la teoría de la abducción de Peirce, como admite y advierte de la falibilidad, se hace cargo de todas ellas.

La abducción tiene lugar, por tanto, in media res y está influida por un contexto previo y una experiencia fundada de la que partir (Duarte, 2018). Es por esto que la abducción no es meramente una intuición, sino que posee una doble naturaleza: lógica, por un lado, y poética, en el sentido de creativa e imaginativa, por otro.

\section{CONSIDERACIONES EN TORNO A LA MEMORIA}

\subsection{La memoria creativa}

Para poder relacionar este tema de la abducción con la creatividad de la memoria tendremos que matizar, primeramente, a qué memoria nos referiremos para delimitar el ámbito de la relación entre creatividad y recuerdos.

Se ha roto con el antiguo modelo de cerebro humano como aparato físico, como un ordenador, metáfora que hacía del cerebro un aparato mecánico en el que las partes no podrían cambiar de forma ni de función. Pero el cerebro humano, como sabemos ahora, dista mucho de ser un ordenador. Como sostiene el psiquiatra Norman Doidge (2008), esta concepción del cerebro derivó en una especie de "nihilismo neurológico" (Doidge, 2008, p. 13), ya que el tratamiento de muchas patologías quedaba en entredicho toda vez que cerebro no podía cambiar y, más aún, no se podía regenerar, solo degeneraba. Pero el cerebro es mucho más complejo y tiene una continua plasticidad. Esto quiere decir que, por supuesto, no existe tal cosa como un desván donde se guardan intactos los recuerdos a los que se recurre como objetos, tal vez un tanto polvorientos, pero que conservan su color, textura y forma inalterados. Como afirma Emilio 
García García (2018), Somos nuestra memoria, pero también podríamos afirmar que la memoria forma parte de todo lo que somos y que, en este sentido, no solo es imprescindible para sostener nuestra identidad, sino que ella misma es identidad. No se trata, pues, de un sustrato definido y unitario que aporta el material del que están hechas nuestras personalidades, sino que es un sustrato neurobiológico no del todo definido, deslocalizado (ver, por ejemplo, Manzanero \& Álvarez, 2015, p. 52). Nuestros cerebros, además, están en continuo proceso de interpretación, de reelaboración y de reconstrucción, en esa dinámica heracliteana de lo vivo.

Por eso me gustaría centrarme en algunos casos específicos dentro del vasto y complejísimo mundo de la memoria para tratar de clarificar algunos aspectos de la misma. Me interesan, particularmente, los casos relacionados con la memoria a largo plazo, declarativa y episódica (ver, por ejemplo, Manzanero \& Álvarez, 2015, pp. 52-56). Esta separación no es más que un recurso académico operacional que no refleja la realidad en toda su extensión. Lo que se ha dado en llamar memoria episódica responde a la idea de recuperación intencionada o consciente de una experiencia previamente aprendida. Tiene que ver con el conocimiento declarativo: aquel cuya demostración se hace por medio del lenguaje. La competencia se manifiesta en declaraciones, es decir, con el saber qué pasó. Por tanto se trata de un proceso deliberado donde esta recuperación consciente implica búsqueda de información y toma de decisiones muy similares a las que se llevan a cabo en el marco abductivo que delimitamos anteriormente. Como afirman Manzanero y Álvarez (2015):

En las tareas de recuerdo intervienen procesos inferenciales similares a los de resolución de problemas como paradigma de procesamiento controlado, opuesto a la recuperación de información mediante el acceso directo a la representación de los estímulos originales. Así pues, las tareas de recuerdo se han considerado tradicionalmente demandantes de recursos. (p. 104).

No se trata, pues, de reproducir el pasado mecánicamente, sino de identificarnos en él, en lo que desempeñará un papel capital la reelaboración y la imaginación. Rememorar, recordar episodios, es una cuestión de realizar viajes mentales constructivos en el tiempo (Michaelian, 2016), y comprenderlo requiere abandonar las teorías filosóficas pasadas sobre la memoria a favor de teorías contraintuitivas, como la teoría de la simulación. Para esta teoría, recordar, estrictamente hablando, no es diferente de imaginar (Michaelian, 2016, p. 237).

De esta forma los procesos inferenciales que se dan en esas tareas de rememoración se oponen a la concepción errónea de un acceso directo a la representación de estímulos originales. Esta recuperación controlada se caracteriza, en definitiva, por la elaboración, y para que esto se lleve a cabo harán falta dos tareas que nos serán familiares: selección entre candidatos (hipótesis) e integración en un contexto perceptivo. En este sentido es en el que hablamos de control o voluntad frente al procesamiento automático que se activa facilitando la consecución de una determinada tarea. En este control interviene la conciencia autonoética (Manzanero \& Álvarez, 2015, p. 115) $\mathrm{y}$, precisamente por ello, tendemos a sobrestimar nuestra capacidad memorística en el sentido de fidelidad a los sucesos originales. Es importante señalar también que el recuerdo episódico produce representaciones que nos llegan, en parte, como representaciones perceptivas, es decir, con contenido sensorial en lugar de proposicional (Michaelian, 2016, p. 54). Sin duda, este aspecto representa también un punto importante a favor de que se tienda a "creer" en los recuerdos con un alto grado de confianza.

Es muy conocida la anécdota que Jean Piaget (1961) relata en La formación del símbolo en el niño sobre la maleabilidad de la memoria:

Si fuese verdadero, uno de mis primeros recuerdos sería cuando tenía dos años. Todavía hoy veo con claridad la siguiente escena, en la que creí hasta los quince. Iba yo sentado en mi cochecito -lo empujaba mi niñera- por los Campos Elíseos, cuando un hombre intentó raptarme. Mientras la niñera se interponía valientemente entre el ladrón y yo, me retuvo el cinturón que me sujetaba. Ella se llevó unos cuantos arañazos, todavía los recuerdo vagamente en su cara. Entonces, se congregó una multitud, se acercó un policía con capa corta y porra blanca y el ladrón puso pies en polvorosa. Todavía veo la escena completa e incluso la localizo cerca de una parada de metro. A los quince, mis 
padres recibieron una carta de mi antigua niñera, en la que contaba que se había hecho del Ejército de Salvación. Quería confesar sus faltas pasadas y, sobre todo, devolver el reloj con el que le habían recompensado en aquella ocasión. Se lo había inventado todo y los arañazos eran de mentira. Por tanto, de pequeño tuve que oír el relato del suceso, que mis padres creyeron, y proyectarlo en el pasado en forma de recuerdo visual. (Loftus \& Ketcham, 2010, p. 47).

Podemos observar en este caso cómo la memoria de Piaget está llena de detalles que parecen confirmar su autenticidad: los arañazos, el policía con capa corta y porra blanca y una parada de metro cercana. Pero el de Piaget no es un caso aislado y peculiar. La mayoría de nosotros, ya adultos, somos capaces de recordar hechos de la infancia: unas vacaciones, un cumpleaños, un accidente, etc., aunque muchos de estos recuerdos infantiles son reconstrucciones, creaciones realizadas a partir del material de otras fuentes de forma no consciente.

De hecho, la memoria en la que estamos interesados no parece estar desarrollada hasta entre los tres y los cinco años, dando lugar a lo que se conoce como amnesia infantil. ¿Significa esto, acaso, que antes de esas edades no tenemos recuerdos? Los niños de edad preescolar podrían almacenar y recuperar hechos autobiográficos, pero estos hechos se almacenan de forma descontextualizada (Silva, Manzanero, \& Contreras, 2016), y con el paso del tiempo y diferentes procesos de elaboración los recuerdos adquieren la forma de una memoria autobiográfica ganando en detalles, coherencia y sentido, en definitiva (Manzanero \& Álvarez, 2015, pp. 231-234). Es decir, que construimos totalidades organizadas en función de un contexto que genera, de forma harto creativa, soluciones a problemas de ambigüedad.

\subsection{Un caso paradigmático y alarmante}

Esta creatividad de nuestra memoria podría pasar por algo anecdótico, curioso y hasta divertido, si, como en el caso de Piaget, adornamos o inventamos ciertas situaciones de la infancia. La demostración de que tenemos algunos recuerdos inventados de una etapa temprana de la vida no nos urge a abandonar el antiguo paradigma de memoria-contenedor. Es más: nuestras experiencias cotidianas parecen confirmar que podemos confiar en nuestra memoria. La alarma con respecto a estos recuerdos inventados surge en ciertos casos extremos. Por ejemplo, en aquellos casos donde personas inocentes han sido condenadas judicialmente debido a testimonios honestos de testigos presenciales "engañados" por sus propios recuerdos. Ahora sí, las devastadoras consecuencias en el ámbito humano que estas situaciones llevan asociadas nos apremian (a nosotros, como sociedad) a desechar los viejos paradigmas y a abrir el camino a una comprensión más amplia y dinámica del funcionamiento de la memoria.

Me gustaría rescatar aquí uno de estos casos extremos que narra Elizabeth Loftus en Juicio a la memoria, testigos presenciales y falsos culpables (Loftus \& Ketcham, 2010) y que me parece paradigmático en dos aspectos. En primer lugar, en relación al componente creativo que estamos abordando, y en segundo lugar, en otra cuestión que se me antoja de sumo interés y que no abordaremos aquí, y es este nuevo juicio de la memoria como sospechosa. Hasta el punto de que Elizabeth Loftus afirma categórica: "somos víctimas inocentes de las manipulaciones de nuestra mente" (Loftus \& Ketcham, 2010, p. 49); como si la memoria fuera llamada a juicio y la jueza hubiera dictado sentencia.

Elizabeth Loftus es una de las psicólogas experimentales que más ha clarificado esta nueva concepción de la memoria. Sus investigaciones sobre los falsos recuerdos son realmente significativas, lo que la ha llevado a dar testimonio como perito en muchos casos judiciales (Loftus, 2018). En este marco, uno de los casos que, según reconoce ella misma, más tristeza le ha causado es del Steve Titus (ver, Loftus \& Ketcham, 2010, pp. 65-101). Steve Titus tuvo que lidiar durante cuatro años con la defensa de su vida y su honor. Nancy, una chica de 17 años que estaba haciendo autostop en Seattle en 1980, fue violada a punta de cuchillo. Tras la denuncia, acompañó a los agentes y señaló el lugar exacto en el que el violador había aparcado su coche azul: había marcas recientes de neumáticos. El investigador, llamado Parker, vio un coche azul aparcado en el exterior de un restaurante cercano al lugar del suceso, en el que Steve Titus y su prometida estaban tomando algo. Veamos de manera esquemática de qué manera inculpaban a Titus los hechos narrados por Nancy y los datos obtenidos a partir de la investigación: 


\begin{tabular}{ll}
\hline $\begin{array}{c}\text { Hechos narrados por Nancy sobre } \\
\text { el agresor y el suceso }\end{array}$ & \multicolumn{1}{c}{ Hechos en torno a Titus } \\
\hline 25-30 años, barba y pelo castaño & Respondía a la descripción \\
$1,80 \mathrm{~m}$ de altura & Menos de 1,70 m de altura \\
Traje color crema & Traje oscuro \\
Coche azul & Coche azul \\
Suceso entre las 18:45 y 19:22 $\mathrm{h}$ & $\begin{array}{l}\text { Salió a las 18:10 } \mathrm{h} \\
\text { Testigo afirma que volvió a las 18:45 } \mathrm{h} \\
\end{array}$ \\
& Llamada registrada a las 19:00 $\mathrm{h}$ \\
\hline
\end{tabular}

\section{Hechos de la investigación / laboratorio}

En el coche y en la ropa de Titus no hallaron nada relacionado con el caso (huellas, fibras, cabellos...)

Los supuestos cabellos del violador recogidos en el jersey de la víctima no coincidían con los de Titus

Las huellas de los neumáticos halladas en el lugar de los hechos no coincidían con las del coche de Titus

Parker seleccionó a seis hombres para el montaje de reconocimiento fotográfico en el que aparentemente no había sesgos: se seleccionaron dos fotos de cada uno de ellos, una de frente y otra de perfil; todos tenían un aspecto normal, con edades entre 25 y 30 años, sin señales ni marcas que los distinguiera; todos ellos tenían el pelo castaño y barba. Tras mostrarle las fotografías a Nancy, ella movió varias veces la cabeza en señal de negación. Parker la instaba a concentrarse, a reflexionar profundamente: "Puede hacer algo", le dijo. Tras un rato de reflexión Nancy señaló las fotografías de la esquina superior derecha de la página señalando a Titus: "Este es el que más se parece".

Desde luego, estamos ante un caso extremo, pero por otro lado puede servir como lente de aumento para ilustrar cómo funciona la memoria también en casos normales, cuando necesitamos dar con la solución a una ambigüedad y se nos presenta un candidato plausible para rellenar ese hueco. De hecho, la prueba más contundente y determinante de la condena de Titus fue la de la identificación por parte de la víctima.
¿Había algún problema con el montaje fotográfico? El abogado defensor de Titus se dio cuenta de que inducía a fijarse en Titus más que en los demás: la foto polaroid de Titus era de menor tamaño, la mitad que las otras cinco fotografías; no había, como en el resto, un reborde negro entre la foto de cara y la de perfil; y el sujeto se reía.

En la vista la víctima ya no tenía ninguna duda de que Titus era el culpable y lo señaló identificándolo sin vacilación. Durante su turno de preguntas el abogado defensor de Titus destacó todas las incoherencias antes señaladas, pero al preguntarle a Nancy si ella seguiría afirmando que fue él quien lo hizo aunque se pudiese demostrar que Steve Titus estaba en otra parte cuando se produjo la violación, ella respondió afirmativamente sin dudarlo un instante. Steve Titus fue declarado culpable de violación en primer grado.

Tras la intervención de un periodista que dedicó meses a investigar este caso se descubrió al verdadero violador, quien, además, fue declarado culpable de tres violaciones más. Nancy acudió a la comisaría a una rueda de reconocimiento. Al ver al verdadero violador por el cristal del espejo se echó a llorar exclamando: “¡Ay, Dios mío! ¡Qué 
le he hecho al señor Titus!". El verdadero violador confesó los hechos y fue condenado. Todo este proceso destrozó la vida de Steve Titus: perdió su trabajo, a su prometida y sus ahorros, y murió de un ataque al corazón con 35 años. Lo que hace esta tragedia aún mayor es que nadie duda de la buena fe de Parker y Nancy.

Este no es un caso aislado, y no solo los estudios llevados a cabo por Elizabeth Loftus demuestran lo maleable que es la memoria: la ONG de Estados Unidos Innocent Project ha conseguido desde 1992, gracias a pruebas de ADN, la excarcelación de 350 presos, algunos de los cuales estaban en el corredor de la muerte. Estas condenas a inocentes se deben a la memoria humana, es decir, declaraciones o identificaciones erróneas por víctimas o testigos, en un 70\% de los $\operatorname{casos}^{1}$ (Loftus \& Ketcham, 2010, p. 17; García García, 2018, p. 83).

Como hemos apuntado anteriormente, este tipo de casos demandan ampliar nuestro conocimiento sobre la memoria. Aquí, la creatividad de la memoria juega un negro papel, en tanto en cuanto esta creatividad no es, ni siquiera, considerada posible.

\section{4. ¿ES LA CREATIVIDAD DE LA MEMORIA UNPROCESOABDUCTIVO?}

En las dos secciones anteriores hemos tratado de describir individualmente la abducción y la memoria episódica. Hemos visto que el razonamiento abductivo es aquel por el cual llegamos a proponer hipótesis, implicando que es a través de la abducción como una nueva idea puede surgir. Por otro lado, las investigaciones más recientes demuestran que la memoria trabaja de manera dinámica. Este funcionamiento de la memoria, en el que aparecen la reconstrucción y la selección de hipótesis, no se ajusta al paradigma que asume el acceso a los recuerdos de manera, podríamos decir, aséptica. En este punto, podemos preguntarnos lo siguiente: si la memoria es, a menudo, creativa y la creatividad es un proceso abductivo, iesta creatividad de la memoria no habría de ser, por tanto, abductiva? Veamos dónde podemos llegar tratando de responder a esta pregunta.

Para ello veamos ahora cómo podemos interpretar la historia de Nancy y Titus a la luz de los rasgos característicos de la abducción:
1. En primer lugar teníamos un enigma, un hueco que rellenar: la víctima se pregunta si alguna de las personas del montaje fotográfico es su agresor. Además, parecía perentorio encontrar la respuesta (Nancy tiene ante sí la posibilidad de cerrar un episodio traumático de su vida) y todo apuntaba a que la solución estaba cerca (Parker ayuda a esta suposición: "Puede hacer algo").

2. En segundo lugar, se produce la conexión con una hipótesis plausible ("Este es el que más se parece") que parece solucionar el puzle planteado. En este punto encontramos el momento de inspiración (el destello abductivo) que equivaldría a cuando el recuerdo, poco a poco, se abre paso. Ya hemos hablado del momento de emoción que se produce en este punto pues uno vislumbra ante sí la solución al enigma; es por este mismo motivo por el que la hipótesis de Nancy (al principio un poco vaga) va ganando credibilidad al no haber otras hipótesis inmediatas que puedan refutarla (Nancy no piensa, por ejemplo, que son dos hombres los que más se parecen al violador). Por tanto, para Nancy su hipótesis ya no es tal, sino un recuerdo.

3. El recuerdo de Nancy se afianza, puesto que, como en las hipótesis abductivas, este se construye atendiendo a principios de verosimilitud de acuerdo al contexto en cuestión. Nancy identifica a Titus con su agresor puesto que hay ciertos rasgos que casan con su descripción. No habría reconocido al agresor en la foto de, pongamos por caso, una mujer o un hombre negro.

4. Sin embargo, no se considera el carácter falible, tentativo y sujeto a nuevos descubrimientos que se da en todo proceso creativo justificado. Precisamente, vemos que el paralelismo con la abducción en los puntos anteriores dota al proceso creativo de la memoria de verosimilitud. Sin embargo, se obvia que es un proceso creativo, una hipótesis. Al final se toma como una verdad prácticamente indubitable porque así es como se puede tomar este tipo de testimonios: como pruebas concluyentes. Esto lleva implícito la concepción de la mente humana como algo que graba acontecimientos como lo hacen los dispositivos digitales: es cierto que podemos admitir los errores en los recuerdos, como lo hace Nancy cuando 
una nueva evidencia se presenta, pero somos reticentes a aceptar el factor falibilidad como algo intrínseco al proceso de recuerdo.

Tenemos aquí el paralelismo entre la reconstrucción de los recuerdos en casos de memoria episódica y los procesos abductivos. Sin embargo, al no reparar en que esta reconstrucción es abductiva se obvia el carácter falible del recuerdo. Vemos, por tanto, cómo el carácter falible del proceso abductivo desaparece cuando es nuestra memoria la que nos proporciona "ideas" (recuerdos) creativos, es decir, no fieles a la realidad

\section{CONCLUSIÓN}

Atendiendo al análisis anterior, podríamos concluir, por tanto, que la creatividad de la memoria debería ser un proceso abductivo. Con esto me refiero a que, ciertamente, sigue los pasos de la abducción incluido el de la falibilidad. Sin embargo, parece que el recuerdo se nos impone, de manera similar a como se nos imponen los juicios perceptivos, y por eso mismo no somos capaces de someterlo a crítica. Entendiendo que existe un perfecto paralelismo entre la generación y selección de hipótesis (abducción) y el proceso de recuperar recuerdos, podemos empezar a analizar estos últimos desde un punto de vista abductivo, lo que implicaría dotarlos de un carácter tentativo y provisional. Esto no debería invalidar nuestra confianza en la memoria, al igual que entender el carácter falible de la abducción no invalida las hipótesis o, por extensión, las teorías científicas: tan solo las hace provisionales hasta que otras mejores aparecen.

En casos tan reglados como el ejemplo anterior, con unas normas de procedimiento tan estrictas como las de un proceso judicial y con consecuencias tan extremas como llevar a un hombre al corredor de la muerte, la afirmación Loftus "somos víctimas inocentes de las manipulaciones de nuestra mente" no resultará extravagante. No obstante, considero que sería torticero y sumamente peligroso trasladar esta conclusión a otros ámbitos. La memoria, como hemos querido mostrar, es creativa, tiene paralelismos con la abducción en el sentido de que está contextualizada, busca rellenar huecos para solucionar enigmas a través de la conexión de elementos que son candidatos a resolver estos puzles. No obstante y esto no la invalida, está sujeta a revisión retractación y mejora, si el caso así lo requiere. No hay nada más engañoso e inhumano que la memoria mecánica o digital. Como señala Kourken Michaelian (2016):

La reacción inicial de uno cuando aprende cómo funciona la memoria (...) podría ser la de desconfiar de la propia memoria. Pero la evolución nos ha equipado con un sistema que es mucho más poderoso y flexible que un simple modelo de almacenamiento sin incurrir, al mismo tiempo, en un descenso profundo de su confiabilidad. En resumen, aunque nuestra imagen intuitiva de la forma en que funciona la memoria puede ser inexacta, nuestra confianza instintiva en nuestros propios recuerdos está justificada. La función de esta memoria simulativa es la de proporcionarnos el conocimiento del pasado. (p. 239, [traducción propia]).

Así, aunque realmente tendamos a sobreestimar la capacidad de la memoria para mantenerse fiel a unos hechos dados y, desde luego, no hay ninguna relación entre confianza y exactitud, como no la hay tampoco en el proceso abductivo, tal vez en otro aspecto tendamos a subestimar su capacidad para constituirnos como lo que somos. La abducción, como la memoria, supone un principio selectivo, un esquema previo donde encajar las piezas que busque cierta coherencia dentro de sus contextos. El olvido, pues, forma parte esencial dentro del funcionamiento de la memoria. Recordar absolutamente todo acabaría produciendo serios problemas. Los que hayan leído la historia de Funes, el memorioso de Borges entenderán lo que digo: Funes no podía olvidar nada. Pero dentro de la literatura científica encontramos también el caso de AJ descrito por Parker, Cahill y McGaugh (2006): AJ, como Funes, no puede olvidar aún cuando quiere hacerlo. Este déficit de olvido es denominado por estos autores síndrome hipertiméstico (Manzanero \& Álvarez, 2015). Igualmente, una de las labores más arduas y complicadas para Salomon Shereshevski, el gran mnemonista tratado y estudiado por Luria, fue aprender el arte de olvidar (Luria, 2009). Como bien dice Borges "pensar es olvidar diferencias, es generalizar, abstraer. En el abarrotado mundo de Funes no había sino detalles" (Borges, 2004, p. 133).

Las memorias de silicio, esos servidores cognitivos externos, nunca podrá sustituir nuestra 
memoria (ver, por ejemplo, Michaelian, 2012) porque, como hemos visto, la memoria humana requiere todo nuestro bagaje para realizar inferencias y fijar así los recuerdos que se constituyen en el cerebro. Perder esta memoria viva supondría perdernos a nosotros mismos, perder nuestra forma de ver, de hacer y de construir lo que nos rodea en el tráfago, muchas veces desordenado y confuso, de este duro oficio que es la vida. 


\section{Referencias}

Aliseda, A. (2005). The logic of abduction in the light of Peirce's pragmatism. Semiotica, 153(1-4), 363-374.

Aliseda, A. (2006). Abductive Reasoning. Logical Investigations into Discovery and Explanation. Dordrecht: Springer.

Barrena, S. (2008). Charles S. Peirce: Razón creativa y educación. Utopía y Praxis Latinoamericana, 40, 11-37.

Borges, J. L. (2004). Ficciones. Barcelona: Ediciones Destino.

Doidge, N. (2008). El cerebro se cambia a sí mismo. Madrid: Aguilar.

Duarte, A. (2018). Abducción y logica docens. Revista de Filosofía, 43(1), 27-47.

Fann, K. T. (1970). Peirce's Theory of Abduction. The Hague, Holland: Martinus Nijhoff.

Gabbay, D., Nossum, R., \& Woods, J. (2006). Context-dependent abduction and relevance. Journal of Philosophical Logic, 35(1), $65-81$.

Gabbay, D. \& Woods, J. (2005). The Reach of Abduction. Insight and Trial. A Practical Logic of Cognitive Systems. Volume 2. Amsterdam: Elsevier.

García García, E. (2018). Somos nuestra memoria. Recordar y olvidar. España: EMSE EDAPP, S. L.

Hintikka, J. (1998). What is Abduction? The Fundamental Problem of Contemporary Epistemology. Transactions of the Charles S. Peirce Society, 34(3), 503-533.

Isaacson, W. (2009). Einstein, su vida y su universo. Barcelona: Debolsillo.

Loftus, E. (2018). Eyewitness science and the legal system. Annual Review of Law and Social Science, 14, 1-10.

Loftus, E. \& Ketcham, K. (2010). Juicio a la memoria. Testigos presenciales y falsos culpables. Barcelona: Alba Oscura.

Luria, A. R. (2009). Pequeño libro de una gran memoria. La mente de un mnemonista. Oviedo: KRK ediciones.

Manzanero, A. L. \& Álvarez, M. Á. (2015). La memoria humana. Aportaciones desde la neurociencia cognitiva. Madrid: Ediciones Pirámide.
Magnani, L. (2015). The eco-cognitive model of abduction,

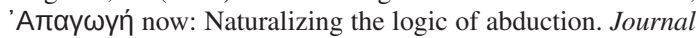
of Applied Logic, 13, 285-315.

Michaelian, K. (2012). Is external memory memory? Biological memory and extended mind. Consciousness and Cognition, $21,1154-1165$

Michaelian, K. (2016). Mental Time Travel: Episodic Memory and Our Knowledge of the Personal Past. Cambridge, MA: MIT Press.

Nubiola, J. (2005). Abduction or the Logic of Surprise. Semiotica, 153(1-4), 117-130.

Parker, E. S., Cahill, L., \& McGaugh, J. L. (2006). A case of unusual autobiographical remembering. Neurocase, 12(1), 35-49.

Peirce, C. S. (1966). The Charles S. Peirce Papers $[32$ rollos de microfilms de los manuscritos conservados en la Houghton Library]. Cambridge: Harvard University Library, Photographic Service.

Peirce, C. S. (2004). The Collected Papers of Charles Sanders Peirce. Electronic Edition, reproducing Vols. I-VI, C. Hartshorne \& P. Weiss (Eds.), Cambridge, MA: Harvard University Press, 1931-1935. Vols. VII-VIII, A. W. Burks (Ed.). Cambridge, MA: Harvard University Press.

Piaget, J. (1961). La formación del símbolo en el niño. México: Fondo de Cultura Económica.

Sagan, C. (1983). Cosmos. Barcelona: Planeta.

Santaella, L. (2005). Abduction: The logic of guessing. Semiotica, 153(1-4), 175-198.

Silva, E. A., Manzanero, A. L., \& Contreras, M. J. (2016). La memoria y el lenguaje en pruebas testificales con menores de 3 a 6 años. Papeles del Psicólogo, 37(3), 224-230.

Walton, D. (2004). Abductive Reasoning. Tuscaloosa, Alabama: The University of Alabama Press.

Walton, D. (2008). The three bases for the enthymeme: A dialogical theory. Journal of Applied Logic, 6(3), 361-379. 


\section{Notas al final}

https://www.innocenceproject.org/ 